\title{
Reviewer Acknowledgements for Global Journal of Health Science, Vol. 8, No. 9
}

Global Journal of Health Science wishes to acknowledge the following individuals for their assistance with peer review of manuscripts for this issue. Their help and contributions in maintaining the quality of the journal are greatly appreciated.

Global Journal of Health Science is recruiting reviewers for the journal. If you are interested in becoming a reviewer, we welcome you to join us. Please find the application form and details at http://recruitment.ccsenet.org and e-mail the completed application form to gjhs@ccsenet.org.

\section{Reviewers for Volume 8, Number 9}

Aedan Simon Olaso

Ahmad Mahran

Aljameel Albandary

Alvin Caballes

Ama Pokuaa Fenny

Angus Nnamdi Oli

Ann M. Thomson

Åsa Premberg

Asad Ali Khan Afridi

Ashley A. Weaver

Ashwini Kucknoor

Azadeh Saki

Bashiru I.I. Saeed

Brian Cook

Daleep Kumar Arora

Darampal Dambhare

David R. Walwyn

Dilek Pirim

Dongxu Fu

Mohammad Biriya

Emad Shdaifat

Enoch Sam

Esteban Ortiz-Prado

Eva Laerkner

Evangelia Mavrikaki

Evanthia Sakellari

Farahnaz Amini

Gabriele Messina

Georgann V. Weissman

Glenda McDonald

Gunta Beta

Helen L. Smits

Ibrahim A. Abdelazim
Isac Silva de Jesus

Jeffery Spickett

Jennifer Johnson

Jillian Kohler

José Joaquín Mira

Kartheek Balapala

Lingzhen Dai

Liu Liu

Liye Suo

Luba Ivanov

Marcel Wullschleger

Mehran Fasihozaman Langerudi

Mohammad Khademlo

Mohsen Barouni

Monica Kvande

Myo Nyein Aung

Nasrin Shahedifar

Nima Hafezi Nejad

Ozra Etemadi

Radu Spataru

Raildo Coqueiro

Raywat Deonandan

Robert Sloan

Rongfang $\mathrm{Hu}$

Samendra Prasad Sherchan

Sandra J. Waters

Satoshi Horiuchi

Seyyed Abdollah Madani

Shaillay Dogra

Srikrishna Ramachandra

Yasam Kemal Akpak

Yongmei Huang

Zohreh Vanaki 